\title{
BAYESIAN APPROACH WITH HIERARCHICAL MARKOV MODELING FOR DATA FUSION IN IMAGE RECONSTRUCTION APPLICATIONS
}

\author{
Ali Mohammad-Djafari \\ Laboratoire des Signaux et Systèmes (CNRS-SUPELEC-UPS) \\ École Supérieure d'Électricité \\ Plateau de Moulon, 91192 Gif-sur-Yvette Cedex, France. \\ E_mail: djafarielss.supelec.fr
}

\begin{abstract}
In many image reconstruction applications, more and more, we need techniques to combine different kind of data. This is the case, for example, in computed tomography $(C T)$ medical imaging where one may use anatomic atlas data with $X$ ray radiographic data or in non destructive testing (NDT) techniques where one wants to use both gamma rays and ultrasound echo-graphic data. In this paper, First we present the basics of Bayesian estimation approach and will see how the compound or hierarchical Markov modeling will give us the necessary tools for data fusion. Then, we present two examples: one in medical imaging CT application and the second in industrial NDT. In both cases, we consider an $X$ ray $C T$ image reconstruction problem using two different kind of data: classical $X$ rays radiographic data and some geometrical informations and propose new methods for these data fusion problems. The geometrical information we use are of two kind: partial knowledge of values in some regions and partial knowledge of the edges of some other regions. We show the advantages of using such informations on increasing the quality of reconstructions. We also show some results to analyze the effects of some errors in these data on the reconstruction results.
\end{abstract}

Keywords: Bayesian data fusion, Compound Markov modeling, Computed tomography, Non destructive testing, Fusion of radiographic and geometric data.

\section{INTRODUCTION}

A widely used technique in industrial non destructive testing (NDT) application is X ray computed tomography (CT). While in medical imaging, classical methods based on back projection (BP) or algebraic reconstruction techniques (ART) give satisfaction, in NDT applications, data acquiring constraints (limited projection angles) are such that these methods do not give satisfactory results. However, in both areas, very often, there is a need for extra information and other kind of data to obtain satisfactory results. Data fusion is then an active area of research in these applications. In this work, we consider the $\mathrm{X}$ ray $\mathrm{CT}$ image reconstruction problem using two different kind of data: classical radiographic (projection) data and some geometrical informations such as partial knowledge of materials in some regions and/or the borders of these or some other regions.

The idea of using geometrical data in CT imaging is not new. Many works on the subject has been done before. See for example $[1,2,3,4,5,6,7,8]$. In $[1,7,8]$, the authors proposed methods for using regions borders from geometrical data in medical imaging and the authors in $[2,3,5,6]$ used the knowledge of some of regions materials. While the application in the first references concerns medical imaging, the application in the second references concerns industrial NDT. But, combining both regions and borders informations from anatomic data is new.

This paper is organized as follows: First, the basics of the Bayesian approach for heterogenous data fusion is presented. Then, we focus on the fusion of X ray and geometrical data and give details of the proposed method. Finally, we present a few simulated experiences showing comparisons of the results using classical back-projection or filtered back-projection methods with those obtained by the proposed method either using or not the geometric data. These results show the advantages of using geometric data when those data are exact and well registered with radiographic data. We also present some preliminary results showing the sensitivity of the proposed method to some errors in geometrical data due to imperfect registration and other uncertainties.

The numerical experiments simulate both a fan beam CT problem in medical imaging applications and a NDT testing of metallic layer shaped media (sandwich structures) such as those of aircraft control surfaces. While, in the first case, we have projection data in $[0,2 \pi]$ angle intervals, in the second case, the structures are such that we cannot have a full range of projection data and the reconstruction problem is a more strongly ill posed one due to limited-angle Radon 
transform null space. The authors in $[3,5]$ has also considered this problem, but the proposed method by these authors are based on projection on convex sets (POCS) which can not account for errors and extra knowledge as easily as in regularization or Bayesian estimation techniques.

\section{BAYESIAN APPROACH FOR DATA FUSION}

Assume that we are observing an unknown body $\boldsymbol{O}$ through two different measurement systems and obtained two sets of data $\boldsymbol{y}$ and $\boldsymbol{z}$. For example, consider a NDT application system where $\boldsymbol{y}$ is a set of $\mathrm{X}$ ray radiographes and $z$ a set of echo-graphic data obtained using a laser or an ultrasound probing system. The $\mathrm{X}$ ray data are related to the mass density $\boldsymbol{x}$ of the matter while the ultrasound data are related to the acoustic reflectivity $\boldsymbol{r}$ of the matter which is more related to the changes of material mass density inside the object and gives more information on the edges of different homogeneous regions. Indeed, assume that, we have linear relations, both between $\boldsymbol{y}$ and $\boldsymbol{x}$ and between $\boldsymbol{z}$ and $\boldsymbol{r}$. Then we have:

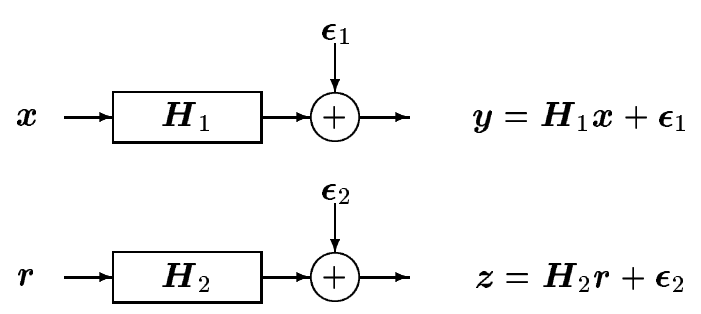

where $\boldsymbol{H}_{1}$ and $\boldsymbol{H}_{2}$ represent, respectively, the linear response matrix of the X-ray CT and ultrasound echo-graphic measurement systems, and $\boldsymbol{\epsilon}_{1}$ and $\boldsymbol{\epsilon}_{2}$ their respective errors (modeling, discretization and measurement errors).

One approach proposed and used by the author [9] and by other collaborators Gautier et al. $[10,6,11]$ is based on a compound or hierarchical Markovian models. In these models, the body object $\boldsymbol{o}$ is assumed to be composed of two or more related quantities $\boldsymbol{o}=(\boldsymbol{x}, \boldsymbol{r})$, where $\boldsymbol{r}$ is related in some way to $\boldsymbol{x}$, for example $r_{j}=g\left(x_{j+1}-x_{j}\right)$, where $g$ can be any monotonic increasing function. In a preceding work [9], we also considered a case, where we had defined $\boldsymbol{o}=(\boldsymbol{x}, \boldsymbol{q}, \boldsymbol{r})$ where the relation between $\boldsymbol{x}$ and $\boldsymbol{r}$ is defined through a hidden edge variable $\boldsymbol{q}$. Here, we give more details for the first case.

Using this model via a Bayesian estimation approach, we can write

$$
p(\boldsymbol{o})=p(\boldsymbol{x}, \boldsymbol{r})=p(\boldsymbol{x} \mid \boldsymbol{r}) p(\boldsymbol{r})
$$

and using the Bayes rule, we have

$$
\begin{aligned}
& p(\boldsymbol{x}, \boldsymbol{r} \mid \boldsymbol{y}, \boldsymbol{z}) \propto \quad p(\boldsymbol{y}, \boldsymbol{z} \mid \boldsymbol{x}, \boldsymbol{r}) p(\boldsymbol{x}, \boldsymbol{r}) \\
& =p(\boldsymbol{y}, \boldsymbol{z} \mid \boldsymbol{x}, \boldsymbol{r}) p(\boldsymbol{x} \mid \boldsymbol{r}) p(\boldsymbol{r}) .
\end{aligned}
$$

To illustrate this approach more in details, we make the following assumptions:
- Conditional independence of $\boldsymbol{y}$ and $\boldsymbol{z}$ :

$$
p(\boldsymbol{y}, \boldsymbol{z} \mid \boldsymbol{x}, \boldsymbol{r})=p(\boldsymbol{y} \mid \boldsymbol{x}) p(\boldsymbol{z} \mid \boldsymbol{r})
$$

- Gaussian process for $\boldsymbol{\epsilon}_{1}$ and $\boldsymbol{\epsilon}_{2}$ which results to:

$$
\begin{array}{ll}
p\left(\boldsymbol{y} \mid \boldsymbol{x} ; \sigma_{1}^{2}\right) \propto & \exp \left[-\frac{1}{2 \sigma_{1}^{2}}\left\|\boldsymbol{y}-\boldsymbol{H}_{1} \boldsymbol{x}\right\|^{2}\right] \\
p\left(\boldsymbol{z} \mid \boldsymbol{r} ; \sigma_{2}^{2}\right) \propto & \exp \left[-\frac{1}{2 \sigma_{2}^{2}}\left\|\boldsymbol{z}-\boldsymbol{H}_{2} \boldsymbol{r}\right\|^{2}\right]
\end{array}
$$

- Markovian model for $\boldsymbol{x} \mid \boldsymbol{r}$ :

$$
p(\boldsymbol{x} \mid \boldsymbol{r}) \propto \exp [-\alpha \phi(\boldsymbol{x} \mid \boldsymbol{r})]
$$

- A non Gaussian model for $\boldsymbol{r}$ :

$$
p(\boldsymbol{r}) \propto \exp [-\beta \psi(\boldsymbol{r})]
$$

Using these assumptions, we obtain

$$
p(\boldsymbol{x}, \boldsymbol{r} \mid \boldsymbol{y}, \boldsymbol{z}) \propto \exp [-J(\boldsymbol{x}, \boldsymbol{r})]
$$

with

$$
\begin{aligned}
J(\boldsymbol{x}, \boldsymbol{r})= & \frac{1}{2 \sigma_{1}^{2}}\left\|\boldsymbol{y}-\boldsymbol{H}_{1} \boldsymbol{x}\right\|^{2}+\frac{1}{2 \sigma_{2}^{2}}\left\|\boldsymbol{z}-\boldsymbol{H}_{2} \boldsymbol{r}\right\|^{2} \\
& +\alpha \phi(\boldsymbol{x} \mid \boldsymbol{r})+\beta \psi(\boldsymbol{r}) .
\end{aligned}
$$

Many schemes can then be proposed to estimate either $\boldsymbol{x}$ or both $(\boldsymbol{x}, \boldsymbol{r})$. We mention here three:

- Simultaneous estimation of both unknowns with the joint MAP estimation (JMAP):

$$
(\widehat{\boldsymbol{x}}, \widehat{\boldsymbol{r}})=\arg \max _{(\boldsymbol{x}, \boldsymbol{r})}\{p(\boldsymbol{x}, \boldsymbol{r} \mid \boldsymbol{y}, \boldsymbol{z})\}
$$

- First estimate $\boldsymbol{r}$ and then use it to estimate $\boldsymbol{x}$ :

$$
\left\{\begin{array}{l}
\widehat{\boldsymbol{r}}=\arg \max _{\boldsymbol{r}}\{p(\boldsymbol{r} \mid \boldsymbol{y}, \boldsymbol{z})\} \\
\widehat{\boldsymbol{x}}=\arg \max _{\boldsymbol{x}}\{p(\boldsymbol{x} \mid \boldsymbol{y}, \widehat{\boldsymbol{r}})\}
\end{array}\right.
$$

where

$$
p(\boldsymbol{r} \mid \boldsymbol{y}, \boldsymbol{z})=\int p(\boldsymbol{x}, \boldsymbol{r} \mid \boldsymbol{y}, \boldsymbol{z}) \mathrm{d} \boldsymbol{x}
$$

and

$$
p(\boldsymbol{x} \mid \boldsymbol{y}, \boldsymbol{r}) \propto p(\boldsymbol{y} \mid \boldsymbol{x}) p(\boldsymbol{x} \mid \boldsymbol{r}) p(\boldsymbol{r}) .
$$

- First estimate $\boldsymbol{r}$ using only $\boldsymbol{z}$ and then use them to estimate $x$ :

$$
\left\{\begin{array}{l}
\widehat{\boldsymbol{r}}=\arg \max _{\boldsymbol{r}}\{p(\boldsymbol{r} \mid \boldsymbol{z})\} \\
\widehat{\boldsymbol{x}}=\arg \max _{\boldsymbol{x}}\{p(\boldsymbol{x} \mid \boldsymbol{y}, \widehat{\boldsymbol{r}})\}
\end{array}\right.
$$

where

$$
p(\boldsymbol{r} \mid \boldsymbol{z}) \propto p(\boldsymbol{z} \mid \boldsymbol{r}) p(\boldsymbol{z}) .
$$

Other schemes are possible [9].

In this paper, we are going to consider only this last scheme which is the easiest and needs least computation. To go further in details we have to choose the prior laws 
$p(\boldsymbol{r})$ and $p(\boldsymbol{x} \mid \boldsymbol{r})$. For $p(\boldsymbol{r})$ we choose a generalized Gaussian law:

$$
p(\boldsymbol{r}) \propto \exp \left[-\alpha \sum_{j}\left|r_{j}\right|^{\beta}\right] \quad \text { with } 1 \leq \beta \leq 2,
$$

to catch the pulse shape nature of $\boldsymbol{r}$ ( $r_{j}$ is almost always equal to zero in homogeneous regions and can take any real value in the borders of these regions). This choice, thanks to long-tailed character of this distribution for the values of $\beta$ near to one, gives the possibility to account for the concentration around zero of the histogram of the values of $r_{j}$ while giving the possibility to have large values. Note that the Gaussian law is a special case for $\beta=2$.

For $p(\boldsymbol{x} \mid \boldsymbol{r})$ we choose a hierarchical Markov model:

$$
p(\boldsymbol{x} \mid \boldsymbol{r}) \propto \exp \left[-q\left(r_{j}\right) \phi\left(x_{j}-x_{j-1}\right)\right]
$$

where $\phi$ is a positive potential function and $q\left(r_{j}\right)$ is a decreasing function to translate the link between the two quantities $\boldsymbol{x}$ and $\boldsymbol{r}$, i.e. when $r_{j}$ is low, it is more probable to be in a homogeneous region and when $r_{j}$ is high, it is more probable to be in a transition region (edges).

Based on these choices, we obtain the following scheme:

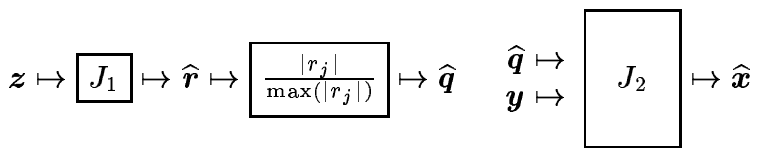

where

$$
\widehat{\boldsymbol{r}}=\arg \max _{\boldsymbol{r}}\{p(\boldsymbol{r} \mid \boldsymbol{z})\}=\arg \min _{\boldsymbol{r}}\left\{J_{1}(\boldsymbol{r} \mid \boldsymbol{z})\right\}
$$

with

$$
J_{1}(\boldsymbol{r} \mid \boldsymbol{z})=\left\|\boldsymbol{z}-\boldsymbol{H}_{2} \boldsymbol{r}\right\|^{2}+\lambda_{1} \sum_{j}\left|r_{j}\right|^{\beta} .
$$

and

$$
\widehat{\boldsymbol{x}}=\arg \max _{\boldsymbol{x}}\{p(\boldsymbol{x} \mid \boldsymbol{y}, \widehat{\boldsymbol{q}})\}=\arg \min _{\boldsymbol{x}}\left\{J_{2}(\boldsymbol{x} \mid \boldsymbol{y} ; \widehat{\boldsymbol{q}})\right\}
$$

with

$J_{2}(\boldsymbol{x} \mid \boldsymbol{y}, \boldsymbol{q})=\left\|\boldsymbol{y}-\boldsymbol{H}_{1} \boldsymbol{x}\right\|^{2}+\lambda_{2} \sum_{j}\left(1-q_{j}\right) \phi\left(x_{j+1}-x_{j}\right)$.

Note that, when the hyperparameters $\lambda_{1}>0$ and $1<\beta<$ 2 are fixed then $J_{1}$ is a convex function of $\boldsymbol{r}$. Then, its optimization can be done by any gradient based algorithm. This will the same for $J_{2}$ if $\phi$ is choosed to be convex and $\lambda_{2}>0$.

\section{FUSION OF GEOMETRIC INFORMATION AND RADIOGRAPHIC DATA}

The previous approach can easily be extended to the CT image reconstruction where we want to include some geometrical information such as partial knowledge of some of the region values and/or some of the positions of edges or region borders. These informations can be obtained, for example, from anatomical atlas in medical imaging or using other measurement systems such as laser or ultrasound echo-graphic data.

The main idea is that, we must use the information we have in those regions, but we must be careful that these informations are subject to uncertainties. Indeed,in those regions we must preserve the given information, but in those regions we do not have information, still we have to use criterion with capabilities of detecting and preserving the edges.

Here, we illustrate an application of the proposed method for the special case of limited angle CT imaging where we have the data $\boldsymbol{y}=\boldsymbol{H}_{1} \boldsymbol{x}+\boldsymbol{\epsilon}$ and we want to include some geometric information in the reconstruction method such as: partial knowledge of borders of different regions given as a map $\boldsymbol{q}$, and partial knowledge of materials in specified regions of the body given as a map $s$ of those values and a map $\boldsymbol{\mu}$ representing the confidence values.

Using some partial knowledge of some regions borders can be considered as a special case of the method proposed in previous section. Actually, in the second step of the proposed method in previous section, we were using the combination of the radiographic data $\boldsymbol{y}$ and $\boldsymbol{q}$ which can be considered as a geometrical (regions borders) data. Here we propose to add a new term to this criterion to include some partial knowledge about the values of pixels in some specified regions. Because this information may be partial and there may also be some uncertainty on it, we use again probabilistic approach and model this through a probability law

$$
p(\boldsymbol{x} \mid \boldsymbol{s}) \propto \exp \left[-\mu_{j} \phi_{2}\left(x_{j}-s_{j}\right)\right]
$$

where $s$ is an image containing the attenuation constant values of some of the regions in the body (not forcibly the same regions for which we know the borders) and $\boldsymbol{\mu}$ an image indicating our degree of confidence about the knowledge of values in those regions ( 0 when no knowledge and 1 when high confidence). Using this model and the discussions in previous sections, the Bayesian MAP estimation approach comes up with the following criterion to optimize to find the an image $\widehat{x}$ which will be the result of fusion of these

$$
\begin{aligned}
J(\boldsymbol{x} \mid \boldsymbol{y}, \boldsymbol{q}, \boldsymbol{s}, \boldsymbol{\mu})= & \left\|\boldsymbol{y}-\boldsymbol{H}_{1} \boldsymbol{x}\right\|^{2} \\
& +\lambda_{1} \sum_{j}\left(1-q_{j}\right) \phi_{1}\left(x_{j+1}-x_{j}\right) \\
& +\lambda_{2} \sum_{j} \mu_{j} \phi_{2}\left(x_{j}-s_{j}\right)
\end{aligned}
$$

Note that, when $\phi_{1}$ and $\phi_{2}$ are choosed to be convex, and when the hyperparameters $\lambda_{1}>0$ and $\lambda_{2}>0$ are fixed and the data $\boldsymbol{y}$ and $\boldsymbol{q}, \boldsymbol{s}$ and $\boldsymbol{\mu}$ are given, this criterion is a convex function of $\boldsymbol{x}$. Then, its optimization can be done by any gradient based algorithm.

A simple choice for $\phi_{1}$ and $\phi_{2}$ is a quadratic $\phi(u)=u^{2}$ or power form $\phi(u)=u^{\beta}$. Other choices are possible, 
for example, all the potential functional forms with edge preserving properties such as

$$
\phi(u)=\left\{2 \ln (\cosh (u)), \quad 2 \sqrt{1+u^{2}}-2\right\}
$$

which are convex $[12,13]$, or

$$
\phi(u)=\left\{\min \left(u^{2}, 1\right), \quad u^{2} /\left(1+u^{2}\right), \quad \ln \left(1+u^{2}\right)\right\}
$$

which are non convex $[14,15,16]$.

The main advantage of choosing convex potential functions with edge preserving properties is to use halfquadratic optimization algorithms $[17,18,19,20]$. In fact, it is now well known that, the joint optimization of a criterion such as $K(\boldsymbol{x}, \boldsymbol{q} \mid \boldsymbol{y})$

$$
\begin{aligned}
K(\boldsymbol{x}, \boldsymbol{q} \mid \boldsymbol{y})= & \left\|\boldsymbol{y}-\boldsymbol{H}_{1} \boldsymbol{x}\right\|^{2} \\
& +\lambda_{1} \sum_{j}\left(1-q_{j}\right)\left(x_{j+1}-x_{j}\right)^{2} \\
& +\lambda_{2} \sum_{j} \psi\left(q_{j}\right)
\end{aligned}
$$

with respect to $(\boldsymbol{x}, \boldsymbol{q})$ is equivalent of the optimization of $J(\boldsymbol{x} \mid \boldsymbol{y})$

$$
\begin{aligned}
J(\boldsymbol{x} \mid \boldsymbol{y})= & \left\|\boldsymbol{y}-\boldsymbol{H}_{1} \boldsymbol{x}\right\|^{2} \\
& +\lambda_{1} \sum_{j} \phi\left(x_{j+1}-x_{j}\right)
\end{aligned}
$$

followed by a straight forward computation of $\boldsymbol{q}$ from $\boldsymbol{x}$, i.e. $q_{j}=g\left(x_{j+1}-x_{j}\right)$ where the expression of $g$ depends on the expression of $\phi$.

We take advantage of this property to propose an iterative algorithm which starts by optimizing (2) with known values of $\boldsymbol{q}$ to obtain $\widehat{\boldsymbol{x}}$, from which we compute a new estimate $\widehat{\boldsymbol{q}}$ using the mentioned property. Then, we replace those values of it by known values of $\boldsymbol{q}$ and use it again in the next iteration.

The whole reconstruction procedure is then the following:

1. Initialize $\boldsymbol{q}=\boldsymbol{q}^{(0)}$

2. Compute $\boldsymbol{x}$ by optimizing the criterion (2);

3. Compute a new value for $\boldsymbol{q}$ from $\boldsymbol{x}$ using the properties of half-quadratic criteria;

4. Replace those a priori known values of $\boldsymbol{q}^{(0)}$ in computed $\boldsymbol{q}$ and return to 2 until convergence.

A final remark concerns the adaptation of the criterion to 2D case where we may distinguish between horizontal, vertical and diagonal edges. In that case we may replace the second term $\lambda_{1} \sum_{j}\left(1-q_{j}\right)\left|x_{j+1}-x_{j}\right|^{\beta_{1}}$ of the criterion (2) by the following:

$$
\begin{aligned}
& \lambda_{h} \sum_{i=1}^{M} \sum_{j=1}^{N-1}\left(1-h_{i j}\right)\left|x_{i, j+1}-x_{i, j}\right|^{\beta_{1}} \\
& \lambda_{v} \sum_{i=1}^{M-1} \sum_{j=1}^{N}\left(1-v_{i j}\right)\left|x_{i+1, j}-x_{i+1, j}\right|^{\beta_{1}} \\
& \lambda_{h v} \sum_{i=1}^{M-1} \sum_{j=1}^{N-1}\left(1-h v_{i j}\right)\left|x_{i+1, j+1}-x_{i, j}\right|^{\beta_{1}} \\
& \lambda_{v h} \sum_{i=1}^{M-1} \sum_{j=2}^{N}\left(1-v h_{i j}\right)\left|x_{i+1, j-1}-x_{i, j}\right|^{\beta_{1}}
\end{aligned}
$$

where $h_{i j}, v_{i j}, h v_{i j}$ and $v h_{i j}$ are, respectively, horizontal, vertical, first diagonal and second diagonal map edges. The corresponding parameters $\lambda_{h}, \lambda_{v}, \lambda_{h v}$ and $\lambda_{v h}$ give the possibility to balance their relative uncertainties.

\section{SIMULATION EXPERIMENTS}

Here, we illustrate an application of the proposed methods for the special case of CT medical imaging. The object is a known numerical phantom in CT which has been proposed by Shepp and Logan [21, 22, 23]. This is a $(256 \times 256)$ image. The sinogram data is obtained by simulating a fan beam tomography with 64 detectors and 128 angular positions over 0 and 360 degrees for the source. The opening angle for the source is 30.4 degrees. The distance between the source and the center of the object is $600 \mathrm{~mm}$ and the dimensions of the reconstructed image is (400 $\mathrm{mm} \times 400 \mathrm{~mm})$.

Figure 1 shows this geometric configuration, the original object and the associated sinogram data.

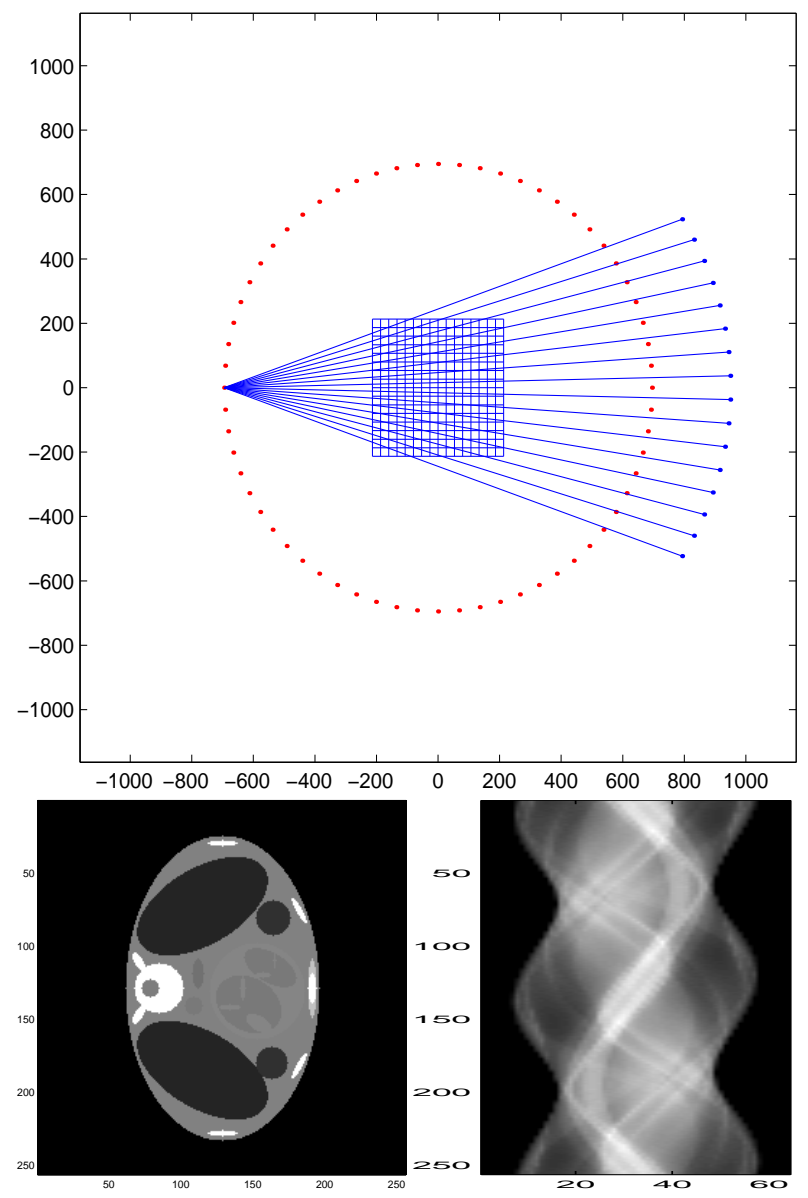

Fig. 1. Geometrical configuration (top), original object (left) and its sinogram data (right). The geometry is a fan beam CT, the object is $(256 \times 256)$ and the sinogram data is $(128 \times 64)$. 


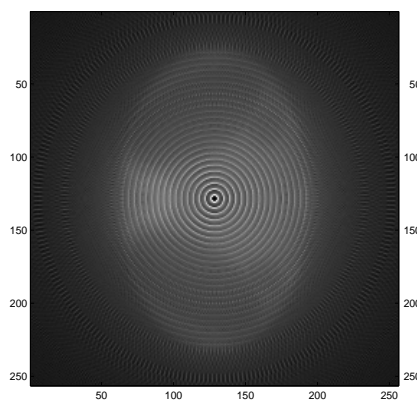

$\mathrm{a}$

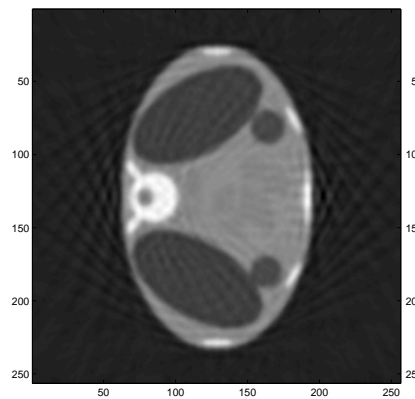

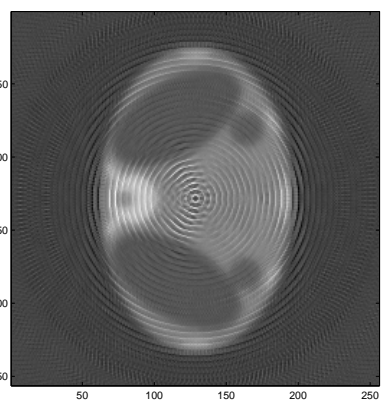

b

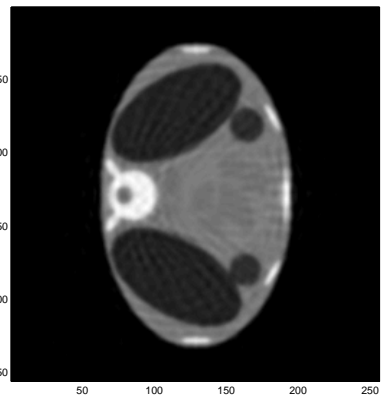

d
Fig. 2. Reconstructions by classical methods: a) backprojection b) filtered back-projection c) quadratic regularization and d) quadratic regularization with positivity constraint.

Figure 2 shows the reconstruction results by classical back-projection or filtered back-projection methods used in commercial scanners. As it is seen on this figure, these results are not satisfactory for the data gathering configuration we proposed where we are looking for a high resolution image $(256 \times 256)$ from a sinogram data which has only $(128 \times 64)$ data points $(64$ detectors and 128 source positions uniformly distributed in $0,2 \pi$ ). We also give here two other results obtained by optimizing the criterion

$$
J(\boldsymbol{x} \mid \boldsymbol{y}, \boldsymbol{q}, \boldsymbol{s})=\left\|\boldsymbol{y}-\boldsymbol{H}_{1} \boldsymbol{x}\right\|^{2}+\lambda_{1} \sum_{j}\left(x_{j+1}-x_{j}\right)^{2}
$$

once over $\mathbf{R}^{n}$ and the second over $\mathbf{R}_{+}^{n}$. In both cases, we used a simple gradient algorithm, but in the second case, we imposed the positivity constraint at each iteration. These results are significantly better than the classical backprojection methods thanks to regularization terms, but they need more computations (approximately two times more computations than a simple back-projection in each iteration).

In the following, the two regularization parameters $\lambda_{1}$ and $\lambda_{2}$ are adjusted empirically and we show the results obtained by this criterion for the following situations:

- when we do not have any geometrical data $(\boldsymbol{q}=\boldsymbol{\mu}=\mathbf{0})$; - when we have only the map of borders $\boldsymbol{q}$ but no region data $(\boldsymbol{\mu}=\mathbf{0})$;

- when we have only the map of regions (characterized by both $\boldsymbol{s}$ and $\boldsymbol{\mu})$ but no other borders data $(\boldsymbol{q}=\mathbf{0})$;

- when we have both the borders $\boldsymbol{q}$ and regions maps $(\boldsymbol{s}, \boldsymbol{\mu})$. - when we have both the borders $\boldsymbol{q}$ and regions maps $(\boldsymbol{s}, \boldsymbol{\mu})$ but with some errors in those data to measure the sensitivity of the results to those errors.

Figure 3 shows the reconstruction results when we assume to know the values of image in three regions (those in white color in the left figure) of the object. Here $\mu_{j}=0$ everywhere excepted the pixels in those three regions.

Figure 4 shows the reconstruction results when we assume to know the borders of some of the regions in the image (those given in the left figure). Here $q_{j}=0 \mathrm{ev}-$ erywhere excepted in the pixels in borders of those three regions where $q_{j}=1$.
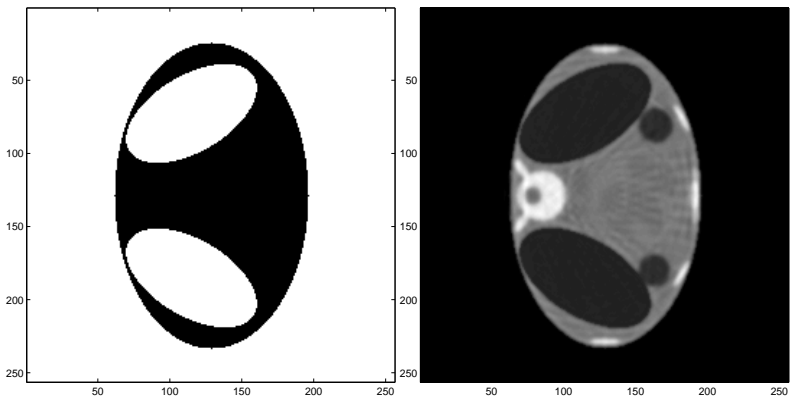

Fig. 3. Reconstructions by data fusion (incomplete known regions): The result in the right is obtained by assuming to know the exact values of densities in the white regions of the figure in the left.
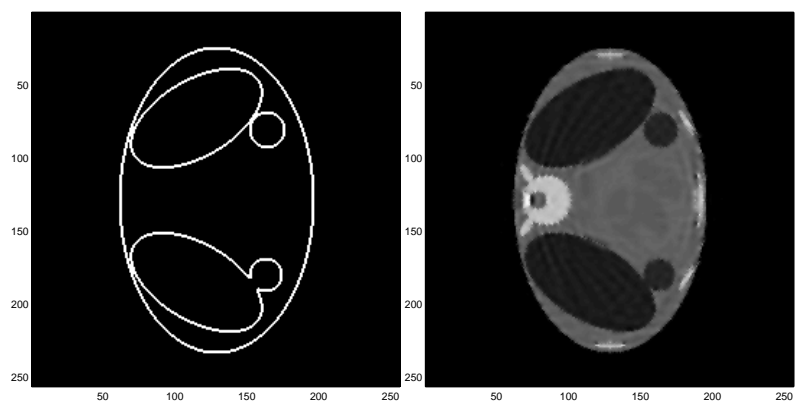

Fig. 4. Reconstructions by data fusion (incomplete regions borders): The result in the right is obtained by assuming to know the borders positions of some of the regions of the object (those in the left figure). 
Figure 5 shows the reconstruction results when we assume to know both the borders of some of the regions and the values of some others (the same as those used in Figures 4 and 5).

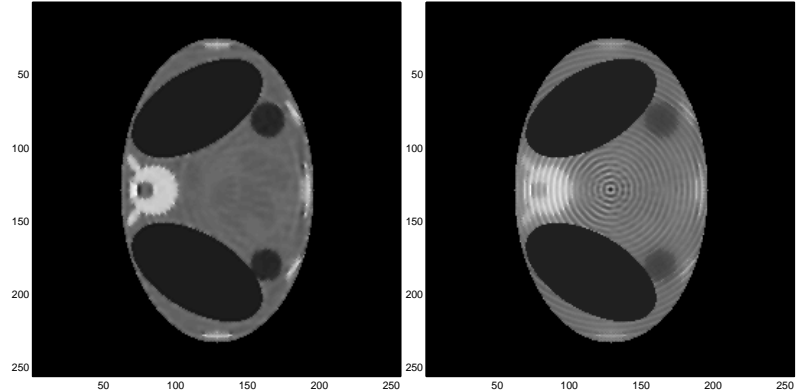

Fig. 5. Reconstructions by data fusion (incomplete regions values, same as in Figure 3, and incomplete regions borders, same as in Figure 4): These results are obtained for two different values of confidence for the regions values (different values of $\lambda_{2}$ which is too low at left but it seems to have good value at right.)

As it is seen from these results, the information content in the knowledge of regions is more valuable than the knowledge of edges only (compare results of Fig. 3 and Fig. 4). Indeed, when we know the values inside a region, in fact we also know its borders. Thus, the extra information in edges in Fig. 5 is not too great. This is the reason for which the results in Fig. 5 are not too different from that of Fig. 3.

However, obviously, fusion of more geometrical information results in more accurate results when the geometrical results are exact. Unfortunately, in practical applications, we need a first step of registration to bring the geometrical informations in the same frames of $\mathrm{X}$ ray radiographic data. In previous simulations, we assumed that this has been done, before starting the reconstruction.

In the following, we give some results to show the sensitivity of the results on some errors of registration. Here we simulated the cases where the geometrical atlas data are obtained with some errors on the orientation of some of the known regions ( 5 degree).

Figure 6 shows the results obtained with these errors in the geometrical data. Comparing these results, we see that the degradations due to these errors are not so crucial if the regularization parameters $\lambda_{1}$ and $\lambda_{2}$ are not too high.

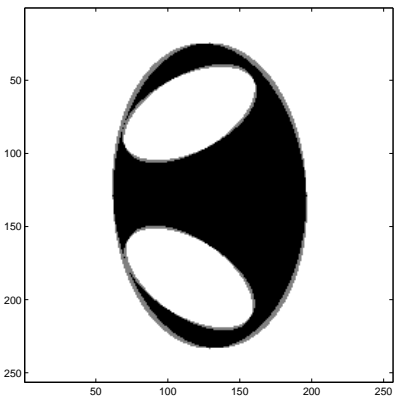

a)

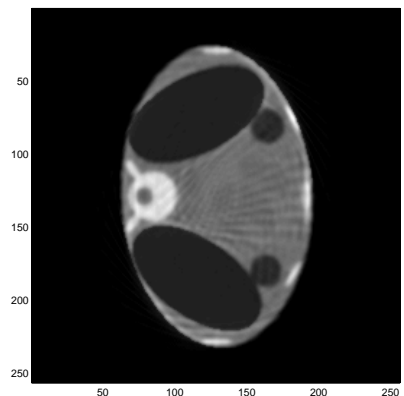

c)

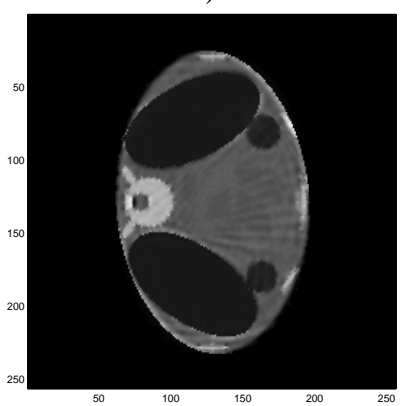

e)

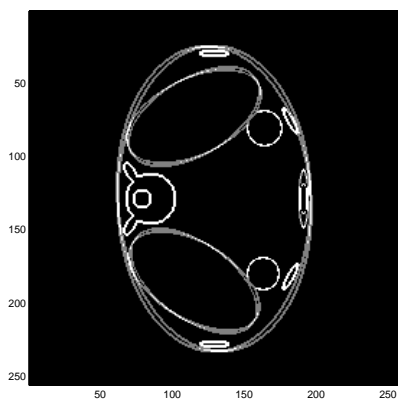

b)

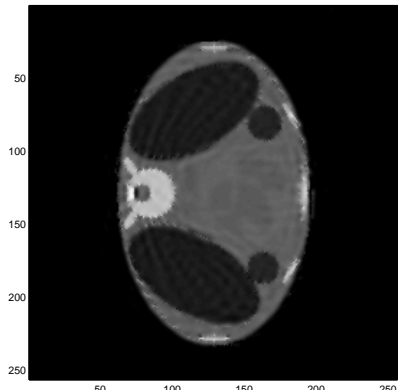

d)

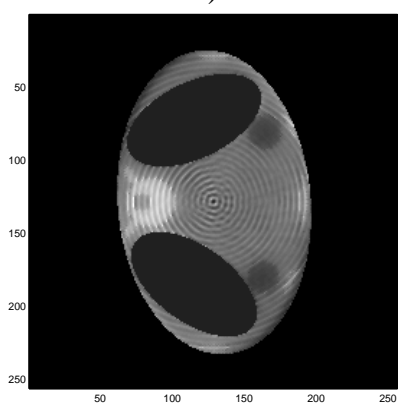

f)
Fig. 6. Reconstructions by data fusion with errors in the geometrical data: a) known regions with errors, b) known borders with errors; c) result using a) to be compared to the result in Fig. 3, d) result using b) to be compared to the result in Fig. 4; e) and f) results using both a) and b) for two different values of confidence for the regions values. These results are to be compared with those of Fig. 5 which were obtained with exact geometrical data. 
All the previous results have been obtained without feedback procedure for re-estimating the unknown edge positions $\boldsymbol{q}$. Figure 7 gives two results which are obtained using the iterative procedure explained at the end of the previous section. Comparing the estimated edges (Fig. 7, right) with the a priori known part of these edges in (Fig. 4, left), we see that new region edges have been estimated. These results are still preliminary. Future studies will focus more on the properties of the mentioned iterative algorithm.
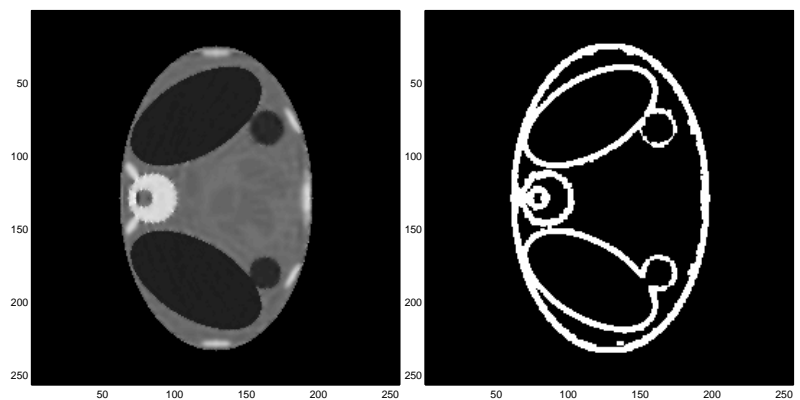

Fig. 7. Reconstructions by incomplete data fusion: This result is obtained using the iterative procedure for reestimating the edges $\boldsymbol{q}$ : On left $\widehat{\boldsymbol{x}}$ (to be compared with the result on Fig. 5) and on right the final re-estimated edges $\widehat{\boldsymbol{q}}$. (to be compared with the known edges on Fig. 4).

\section{CONCLUSIONS}

We proposed a Bayesian approach for data fusion in inverse problems which arise in medical or NDT imaging systems. We illustrated how a hierarchical Markov model can be useful to these data fusion problems where one set of data gives more information about the regions volume (For example X-rays) and another set of data gives information about the regions edges (such as laser or ultrasound echographic data). Then, we considered a medical imaging CT application example and showed how the proposed method can be used to include some anatomical information in the reconstruction problem. We used two kind of geometrical information: partial knowledge of values in some regions and/or partial knowledge of the borders of some other regions. We showed the advantages of using such informations on increasing the quality of reconstructions. We also showed some results to analyze the effects of some errors in anatomic data on the reconstructed results. Finally, we proposed an iterative algorithm which starts by using the available knowledge of edges and regions to compute a first estimate of the image from which we re-estimate the edges and reuse them again to increase more and more the quality of reconstructions.

\section{REFERENCES}

[1] G. Gindi, M. Lee, A. Rangarajan, and I. G. Zubal, "Bayesian reconstruction of functional images using anatomical information as priors," IEEE Trans. Medical Imaging, vol. MI-12, no. 4, pp. 670-680, 1993.

[2] M. Fiani, J. Idier, and S. Gautier, "Algorithmes ART semi-quadratiques pour la reconstruction à partir de radiographies," in Actes du $18^{\mathrm{e}}$ colloque GRETSI, Toulouse, Sept. 2001.

[3] J. Boyd and J. Little, "Complementary data fusion for limited-angle tomography," in Proceeding of Computer Vision and Pattern Recognition 1994, Seattle, WA, USA, 1994, pp. 288-294.

[4] G. Matsopoulos, S. Marshall, and J. Brunt, "Multiresolution morphological fusion of $\mathrm{mr}$ and ct images of the human brain," IEE Proceedings on Vision, Image and Signal Processing, vol. 141 Issue: 3, pp. 137 $142,1994$.

[5] J. Boyd, "Limited-angle computed tomography for sandwich structures using data fusion," Journal of Nondestructive Evaluation, vol. 14, no. 2, pp. 61-76, 1995.

[6] S. Gautier, G. Le Besnerais, A. Mohammad-Djafari, and B. Lavayssière, "Fusion de données radiographiques et ultrasonores, en vue d'une applicaion en contrôle non destructif," Clermont-Ferrand, May 1995, Second international workshop on inverse problems in electromagnetism and acoustic.

[7] S. Som, M. Braun, and B. Hutton, "Incorporation of anatomical priors in spect reconstruction.," in Joint Workshop: Emerging Computational Methods for Application of SPECT in Cardiology, Neurology and Oncology, Utrecht, Netherland, 1998.

[8] S. Som, B. Hutton, and M. Braun, "Edge-preserving regularization based on combined anatomical and functional data," in Joint Workshop: Emerging Computational Methods for Application of SPECT in Cardiology, Neurology and Oncology, Utrecht, Netherland, 1998.

[9] A. Mohammad-Djafari, "Probabilistic methods for data fusion," in Maximum Entropy and Bayesian Methods, Boise, ID, Aug. 1997.

[10] S. Gautier, G. Le Besnerais, A. Mohammad-Djafari, and B. Lavayssière, Data fusion in the field of non destructive testing, Maximum Entropy and Bayesian Methods. Kluwer Academic Publ., Santa Fe, NM, K. Hanson edition, 1995.

[11] S. Gautier, Fusion de données gammagraphiques et ultrasonores. Application au contrôle non destructif, Phd thesis, Université de Paris-Sud, Orsay, Dec. 1996. 
[12] P. J. Green, "Bayesian reconstructions from emission tomography data using a modified EM algorithm," IEEE Trans. Medical Imaging, vol. MI-9, no. 1, pp. 84-93, Mar. 1990.

[13] P. Charbonnier, L. Blanc-Féraud, G. Aubert, and M. Barlaud, "Two deterministic half-quadratic regularization algorithms for computed imaging," in Proc. IEEE ICIP, Austin, TX, USA, Nov. 1994, vol. 2, pp. 168-172.

[14] A. Blake and A. Zisserman, Visual reconstruction, The MIT Press, Cambridge, 1987.

[15] T. Hebert and R. Leahy, "A generalized EM algorithm for 3-D Bayesian reconstruction from Poisson data using Gibbs priors," IEEE Trans. Medical Imaging, vol. 8, no. 2, pp. 194-202, June 1989.

[16] D. Geman and G. Reynolds, "Constrained restoration and the recovery of discontinuities," IEEE Trans. Pattern Anal. Mach. Intell., vol. 14, no. 3, pp. 367-383, Mar. 1992.

[17] D. Geman and C. Yang, "Nonlinear image recovery with half-quadratic regularization," IEEE Trans. Image Processing, vol. 4, no. 7, pp. 932-946, July 1995.

[18] G. Aubert and L. Vese, "A variational method in image recovery," SIAM J. Num. Anal., vol. 34, no. 5, pp. 1948-1979, Oct. 1997.

[19] P. Charbonnier, L. Blanc-Féraud, G. Aubert, and M. Barlaud, "Deterministic edge-preserving regularization in computed imaging," IEEE Trans. Image Processing, vol. 6, no. 2, pp. 298-311, Feb. 1997.

[20] J. Idier, Y. Goussard, and A. Ridolfi, "Unsupervised image segmentation using a telegraph parameterization of Pickard random fields," in Spatial statistics. Methodological aspects and some applications, M. Moore, Ed., vol. 159 of Lecture notes in Statistics, pp. 115-140. Springer Verlag, New York, NY, 2001.

[21] B. F. Logan and L. A. Shepp, "Optimal reconstruction of a function from its projections," Duke Math. J., vol. 42, pp. 645-659, 1975.

[22] L. A. Shepp and Y. Vardi, "Maximum likelihood reconstruction for emission tomography," IEEE Trans. Medical Imaging, vol. MI-1, pp. 113-122, 1982.

[23] G. T. Herman, Image reconstruction from projections. The fundamentals of computerized tomography, Academic Press, New York, 1980. 